\title{
Antibiotic Susceptibility Pattern of Nosocomial Isolates of Staphylococcus aureus in a Tertiary Care Hospital, Nepal
}

\author{
Shrestha B, ${ }^{1}$ Pokhrel BM, ${ }^{2}$ Mohapatra TM ${ }^{1}$ \\ 'Department of Microbiology, Institute of Medical Sciences, Banaras Hindu University, Varanasi, India, \\ ${ }^{2}$ Department of Microbiology, Institute of Medicine, Tribhuvan University, Kathmandu, Nepal.
}

\section{ABSTRACT}

Introduction: Methicillin resistant Staphylococcus aureus (MRSA), the most common cause of nosocomial infection has been a major cause of morbidity and mortality around the world. They are normally resistant to most of the antibiotics used in clinical practice. This study has been carried out to find out the resistance pattern among $S$. aureus.

Methods: During November 2007 to June 2008, clinical samples from patients with nosocomial infection were processed for culture and sensitivity following standard methodology in microbiology laboratory, Tribhuvan University teaching hospital, Kathmandu, Nepal.

Results: Among 149 Staphylococcus aureus isolates, highest resistance was observed against Penicillin (91.94\%) followed by Fluoroquinolone (61.74\%), Erythromycin (52.94\%), Gentamicin (46.98\%), Cotrimoxazole $(42.95 \%)$, Tetracycline $(40.94 \%)$ and others, whereas susceptibility was observed maximum against Chloramphenicol (94.85\%) followed by Rifampicin (92.61\%), Tetracycline $(59.06 \%)$, Cotrimoxazole $(57.04 \%)$, and others. None of the isolates were resistant to Vancomycin and Teicoplanin. Of these isolates $44.96 \%$ of the isolates were Methicillin resistant S. aureus (MRSA). Resistance to Penicillin, Fluoroquinolone, Erythromycin, Gentamicin, Co-trimoxazoleand Tetracycline were associated significantly with MRSA isolates $\left(X^{2}=8.779, p<0.05, X^{2}=74.233, p<0.05, X^{2}=84.2842\right.$, $\mathrm{p}<0.05, \mathrm{X}^{2}=108.2032, \mathrm{p}<0.05, \mathrm{X}^{2}=88.1512, \mathrm{p}<0.05$ and $\mathrm{X}^{2}=79.1876, \mathrm{p}<0.05$ respectively). Although most of the Methicillin sensitive $S$. aureus (MSSA) isolates were susceptible to both Rifampicin and Chloramphenicol, only Rifampicin susceptibility was significantly associated with them $\left(\mathrm{X}^{2}=\right.$ $10.1299, \mathrm{p}<0.05)$. Among three Biochemical tests for the detection of $\beta$ lactamase detection namely chromogenic, iodometric and acidimetric test, chromogenic test method had highest sensitivity and specificity.

Conclusions: Since MRSA comprised a greater part of S. aureus isolates and were multi-resistant, patients infected by such strains should be identified and kept in isolation for hospital infection control and treated with second line of drug like vancomycin.

Key Words: $\beta$ lactamase, methicillin resistant Staphylococcus aureus, methicillin sensitive Staphylococcus aureus, resistance pattern

\author{
Correspondence: \\ Mrs. Bidya Shrestha \\ Institute of Medical Science \\ Banaras Hindu University \\ Varanasi, India. \\ Email: b_shrestha_07@hotmail.com \\ Phone: 977-1-5522788
}


Shrestha et al. Antibiotic Susceptibility Pattern of Nosocomial Isolates of Staphylococcus aureus ...

\section{INTRODUCTION}

Staphylococcus aureus acquired resistance to Methicillin soon after its introduction in therapy. These strains called Methicillin resistant $S$. aureus (MRSA) are a major cause of morbidity and mortality around the world and have been a most common cause of nosocomial infection since late 1970s. ${ }^{1}$ After acquisition of Methicillin resistance in $S$. aureus, there has been a steady rise in the prevalence of MRSA in many countries.

The prevalence rate of MRSA had approached to $50 \%$ in the US hospitals. ${ }^{2}$ In the UK, $44 \%$ of $S$. aureus isolated from health care system were MRSA and in Japan 60$70 \%$ of $S$. aureus were MRSA in inpatients. ${ }^{3,4}$ It has been stated that hospital associated MRSA are often multiple resistant to other commonly used antibiotics while, community acquired MRSA are often resistant only to $\beta$ lactam antibiotics and Erythromycin. ${ }^{5}$ The aim of this study was to find out the prevalence of nosocomial MRSA in a tertiary care center Kathmandu.

\section{METHODS}

A prospective descriptive study was conducted in Tribhuvan University teaching hospital, Kathmandu, Nepal during November 2007 to June 2008. Consecutive clinical samples submitted in microbiology department for culture and sensitivity from patients with nosocomial infection were processed and inoculated onto chocolate agar (CA), blood agar (BA) and MacConkey agar (MA) as required by following standard methodology ${ }^{6}$ The $S$. aureus isolates $(n=149)$ were identified on the basis of Gram's reaction, biochemical tests and agglutination test. Gram positive cocci in clusters and in short chains, catalase positive, oxidase negative, fermentative, Voges Proskauer positive, mannitol fermenter, clumping factor positive, DNase positive, coagulase positive and Staphytect plus latex agglutination (Oxoid, UK) positive were identified as $S$. aureus.

Antibiotic sensitivity test was performed by disc diffusion test following Kirby Bauer method. ${ }^{7}$ Antibiotic discs (Oxoid, UK) recommended by Food and Drug administration (FDA) were used. ${ }^{7}$ The antibiotics Penicillin (10 unit), Oxacillin (1 $\mu \mathrm{g})$, Cefoxitin (30 $\mu \mathrm{g})$, Cotrimoxazole $(1.25 / 23.75 \mu \mathrm{g})$, Rifampicin (5 $\mu \mathrm{g})$, Gentamicin (10 $\mu \mathrm{g})$, Tetracycline (30 $\mu \mathrm{g})$, Chloramphenicol (30 $\mu \mathrm{g})$, Erythromycin (15 $\mu \mathrm{g})$, Ciprofloxacin $(5 \mu \mathrm{g})$, Vancomycin $(30 \mu \mathrm{g})$, Teicoplanin $(30 \mu \mathrm{g})$, Norfloxacin $(10 \mu \mathrm{g})$ and Nitrofurantoin (300 $\mu \mathrm{g})$ were used. In urine and urinary catheter isolates, Erythromycin, Chloramphenicol and Ciprofloxacin were not used and Novobiocin (5 $\mu \mathrm{g}$ for identification), Norfloxacin and Nitrofurantoin were used.

Isolates resistant to both Oxacillin and Cefoxitin were identified as MRSA and those susceptible were identified as Methicillin sensitive $S$. aureus (MSSA).

$\beta$ lactamase test was performed by three biochemical methods namely chromogenic method, acidimetric method and iodometric method on the colony growing around the penicillin disc. ${ }^{6,8}$ For chromogenic test method, Nitrocefin disc (BBL, USA) was used and reagents for acidimetric and iodometric method were prepared in house.

S. aureus ATCC 43300 and S. aureus ATCC 25923 were used as MRSA and MSSA reference strains. Chi square test was used for the statistical analysis of the data.

The study was approved by the institutional review board, TUTH.

\section{RESULTS}

Among 149 nosocomial $S$. aureus isolates (including 13 isolates from urine and urinary catheter), highest resistance was observed against Penicillin (91.94\%) followed by Fluoroquinolone, Erythromycin, Gentamicin, Cotrimoxazole and Tetracycline (Table 1). On the other hand, susceptibility was observed maximum against Chloramphenicol (94.85\%) followed by Rifampicin, Tetracycline and Cotrimoxazole. None of the isolates were resistant to glycopeptides Vancomycin and Teicoplanin.

Of 149 isolates $44.96 \%(n=67)$ isolates were resistant to both Oxacillin and Cefoxitin and hence were identified as MRSA. Obviously 82 isolates were susceptible to these antibiotics and were identified as MSSA.

A uniform pattern of antibiotic susceptibility/ and resistance was observed among the 38 MRSA isolates. They exhibited a uniform pattern of resistance to Cotrimoxazole, Fluoroquinolone, Gentamicin, Tetracycline and Erythromycin; and susceptibility to Rifampicin and Chloramphenicol. Similarly out of 82 MSSA isolates, 41 isolates exhibited a uniform pattern of susceptibility to Cotrimoxazole, Fluoroquinolone, Gentamicin, Tetracycline, Erythromycin, Rifampicin and Chloramphenicol.

Among MRSA isolates cent percent isolates were resistant to Penicillin and Fluoroquinolone showing their association with these antibiotic resistance $\left(X^{2}=8.77\right.$, $\mathrm{P}<0.05$ and $\mathrm{X}^{2}=74.23, \mathrm{P}<0.05$ respectively). Most of the MRSA isolates were resistant to Erythromycin, Gentamicin, Co-trimoxazole and Tetracycline showing significant association of MRSA with resistance to these antibiotics $\left(X^{2}=84.28, P<0.05, X^{2}=108.20\right.$, $P<0.05, X^{2}=88.15, P<0.05$ and $X^{2}=79.18, P<0.05$ respectively) (Table 1 ). 
Shrestha et al. Antibiotic Susceptibility Pattern of Nosocomial Isolates of Staphylococcus aureus ...

Table 1. Susceptibility of isolates to different antibiotics

\begin{tabular}{|c|c|c|c|c|c|c|c|c|}
\hline \multirow{2}{*}{ Antibiotic } & \multirow{2}{*}{$\mathbf{R} / \mathbf{S}$} & \multicolumn{2}{|c|}{ MRSA } & \multicolumn{2}{|c|}{ MSSA } & \multicolumn{2}{|c|}{ Total } & \multirow{2}{*}{$\begin{array}{c}\text { Grand } \\
\text { total }\end{array}$} \\
\hline & & No. & $\%$ & No. & $\%$ & No & $\%$ & \\
\hline \multirow{2}{*}{ Penicillin } & $\mathrm{R}$ & 67 & 100 & 70 & 85.36 & 137 & 91.94 & \multirow{2}{*}{149} \\
\hline & S & 0 & 0 & 12 & 14.63 & 12 & 8.05 & \\
\hline \multirow{2}{*}{ Erythromycin } & $\mathrm{R}$ & 60 & 95.23 & 12 & 16.43 & 72 & 52.94 & \multirow{2}{*}{136} \\
\hline & S & 3 & 4.76 & 61 & 83.56 & 64 & 47.05 & \\
\hline \multirow{2}{*}{ Cotrimoxazole } & $\mathrm{R}$ & 57 & 85.07 & 7 & 8.53 & 64 & 42.95 & \multirow{2}{*}{149} \\
\hline & $\mathrm{S}$ & 10 & 14.92 & 75 & 91.46 & 85 & 57.04 & \\
\hline \multirow{2}{*}{ Chloramphenicol } & $\mathrm{R}$ & 5 & 7.93 & 2 & 2.73 & 7 & 5.14 & \multirow{2}{*}{136} \\
\hline & $\mathrm{S}$ & 58 & 92.06 & 71 & 97.26 & 129 & 94.85 & \\
\hline \multirow{2}{*}{ Fluoroquinolone } & $\mathrm{R}$ & 67 & 100 & 25 & 30.48 & 92 & 61.74 & \multirow{2}{*}{149} \\
\hline & $\mathrm{S}$ & 0 & 0 & 57 & 69.51 & 57 & 38.25 & \\
\hline \multirow{2}{*}{ Rifampicin } & $\mathrm{R}$ & 10 & 14.72 & 1 & 1.22 & 11 & 7.38 & \multirow{2}{*}{149} \\
\hline & S & 57 & 85.07 & 81 & 98.78 & 138 & 92.61 & \\
\hline \multirow{2}{*}{ Gentamicin } & $\mathrm{R}$ & 63 & 94.02 & 7 & 8.53 & 70 & 46.98 & \multirow{2}{*}{149} \\
\hline & $S$ & 4 & 5.97 & 75 & 91.46 & 79 & 53.02 & \\
\hline \multirow{2}{*}{ Tetracycline } & $\mathrm{R}$ & 54 & 80.59 & 7 & 8.53 & 61 & 40.94 & \multirow{2}{*}{149} \\
\hline & $\mathrm{S}$ & 13 & 19.40 & 75 & 91.46 & 88 & 59.06 & \\
\hline \multirow{2}{*}{ Nitrofurantoin } & $\mathrm{R}$ & 0 & 0 & 0 & 0 & 0 & 0 & \multirow{2}{*}{13} \\
\hline & S & 4 & 100 & 9 & 100 & 13 & 100 & \\
\hline
\end{tabular}

On the contrary, most of the MSSA isolates were susceptible to Rifampicin and Chloramphenicol but only Rifampicin susceptibility was associated with MSSA $\quad\left(X^{2}=10.12, \quad P<0.05\right)$. Neither MRSA nor MSSA showed any predilection towards susceptibility to Chloramphenicol, therefore susceptibility was not associated with any of them $\left(X^{2}=1.87, P>0.05\right)$. All 13 urine and urinary catheter isolates were susceptible to Nitrofurantoin.

Most MRSA isolates from all infection sites were multi-resistant (Table 2). Very few MRSA isolates were susceptible to the tested antibiotics except for Chloramphenicol and Rifampicin. Whereas, most of MSSA were susceptible to most of the FDA recommended antibiotics. ${ }^{7}$ Penicillin resistance was observed the most among MSSA (Table 3).

$\beta$ lactamase test by all three test methods almost correlated well with the susceptibility pattern to Penicillin. One penicillin resistant MRSA isolate gave negative result with all three tests. Of 137 Penicillin resistant isolates, 1, 2 and 3 isolates gave false negative result with chromogenic, acidimetric and iodometric tests respectively. Similarly, of 12 penicillin susceptible isolates 1,1 and 2 isolates gave false positive result with chromogenic, acidimetric and iodometric tests respectively. The sensitivity of chromogenic, acidimetric and iodometric test was obtained as $99.27 \%, 98.54 \%$ and $97.81 \%$ respectively. Similarly specificity of chromogenic, acidimetric and iodometric was $91.66 \%$, $91.66 \%$ and $83.33 \%$ respectively. Therefore, among three tests, chromogenic test method was found to have highest sensitivity and specificity.

\section{DISCUSSION}

Small percent (8\%) of the nosocomial S. aureus isolates were susceptible to Penicillin which was in concordance with the reported $10 \%$ in USA. ${ }^{5}$ Greater susceptibility to Chloramphenicol was observed without any predilection either to MRSA or to MSSA in present study. Chloramphenicol used to be a very effective antibiotic and its overwhelming use in clinical practice in past has led to emergence of resistance due to which its use has been discontinued. ${ }^{9}$ Later as suggested by present finding, the organisms have lost resistance to this antibiotic thus rendering them susceptible to Chloramphenicol in these days.

Most MSSA isolates were susceptible to almost all of the FDA recommended antibiotics. On the contrary, most MRSA isolates were resistant to most of the FDA recommended antibiotics similar to what has been stated in USA. ${ }^{5}$ In present study probably the first of its kind, the prevalence of nosocomial MRSA isolates obtained 
Table 2. Susceptibility pattern of MRSA

\begin{tabular}{lcccccccccc}
\hline Isolate & Peni & Eryth & cotri & chlo & Cipr & Rifa & Genta & Tetra & Norfl & Nitr \\
\hline Pus & 41 & $40(1)$ & $35(6)$ & $3(38)$ & 41 & $4(37)$ & $40(1)$ & $33(8)$ & - & - \\
Abscess & 1 & 1 & 1 & 1 & 1 & $(1)$ & 1 & 1 & - & - \\
W swab & 3 & $2(1)$ & $2(1)$ & $(3)$ & 3 & $(3)$ & 3 & $2(1)$ & - & - \\
Bed sor & 1 & 1 & 1 & $(1)$ & 1 & 1 & 1 & 1 & - & - \\
Sputum & 10 & $9(1)$ & $9(1)$ & $(10)$ & 10 & $3(7)$ & $9(1)$ & $8(2)$ & - & - \\
Endo T & 2 & 2 & 2 & $(2)$ & 2 & $(2)$ & $1(1)$ & $1(1)$ & - & - \\
Tra asp & 2 & 2 & 2 & $(2)$ & 2 & $1(1)$ & 2 & 2 & - & - \\
Urine & 2 & - & $1(1)$ & - & - & $(2)$ & $1(1)$ & 2 & 2 & $(2)$ \\
U cathe & 2 & - & 2 & - & - & $(2)$ & 2 & 2 & 2 & $(2)$ \\
Others & 3 & $3(1)$ & $2(1)$ & $1(2)$ & 3 & $1(2)$ & 3 & $2(1)$ & - & - \\
Total & 67 & $60(3)$ & $57(10)$ & $5(58)$ & 63 & $10(57)$ & $63(4)$ & $54(13)$ & 4 & $(4)$ \\
Grand Total & 67 & 63 & 67 & 63 & 63 & 67 & 67 & 67 & 4 & $(4)$ \\
\hline
\end{tabular}

Numbers in parenthesis are the number of susceptible isolates

W Swab: wound swab, Bed sor: bed sore, Endo T: endotracheal tube, Tra Asp: tracheal aspirate, U cathe: urinary catheter.

Peni: penicillin, Eryth: erythromycin, Cotri: cotrimoxazole, Chlo: chloramphenicol, Cipr: ciprofloxacin, Rifa: rifampicin, Genta: gentamicin, Tetra: tetracycline, Norfl: norfloxacin, Nitr: nitrofurantoin

Table 3. Susceptibility pattern of MSSA

\begin{tabular}{|c|c|c|c|c|c|c|c|c|c|c|}
\hline \multirow[t]{2}{*}{ Isolates } & \multicolumn{10}{|c|}{ Antibiotics } \\
\hline & Peni & Eryth & Cotri & Chlo & Cipr & Rifa & Genta & Tetra & Norfl & Nitr \\
\hline Pus & $49(6)$ & $11(44)$ & $6(49)$ & $2(53)$ & 17 (38) & $1(54)$ & $6(49)$ & $6(49)$ & & \\
\hline Abscess & $2(2)$ & $1(3)$ & (4) & (4) & $1(3)$ & (4) & (4) & (4) & & \\
\hline W swab & 2 & (2) & (2) & (2) & (2) & (2) & (2) & $1(1)$ & & \\
\hline Ear dis & 1 & (1) & (1) & (1) & (1) & (1) & (1) & (1) & & \\
\hline Sputum & 3 & (3) & $1(2)$ & (3) & $1(2)$ & (3) & (3) & (3) & & \\
\hline Urine & $3(1)$ & & (4) & & & (4) & (4) & (4) & $3(1)$ & (4) \\
\hline U cathe & 5 & & (5) & & & (5) & (5) & (5) & $3(2)$ & (5) \\
\hline Others & $5(3)$ & (8) & (8) & (8) & (8) & (8) & $1(7)$ & (8) & & \\
\hline Total & $70(12)$ & $12(61)$ & 7 (75) & $2(71)$ & $19(54)$ & $1(81)$ & 7 (75) & $7(75)$ & $6(3)$ & (9) \\
\hline Grand Total & 82 & 73 & 82 & 73 & 73 & 82 & 82 & 82 & 9 & 9 \\
\hline
\end{tabular}

Numbers in parenthesis are the number of susceptible isolates

W Swab: wound swab, Bed sor: bed sore, Endo T: endotracheal tube, Tra Asp: tracheal aspirate, U cathe: urinary catheter

Peni: penicillin, Eryth: erythromycin, Cotri: cotrimoxazole, Chlo: chloramphenicol, Cipr: ciprofloxacin, Rifa: rifampicin, Genta: gentamicin, Tetra: tetracycline, Norfl: norfloxacin, Nitr: nitrofurantoin

is, though low compared to $77 \%$ reported in Taiwan in 2001 , the prevalence is alarming. ${ }^{10}$ Patients from all over the nation seek care in this tertiary care hospital and referral cases make a great percent of its patients. The prevalence of MRSA could have become high due to the movement of patients from hospital to hospital and finally landing in this hospital. These nosocomial MRSA strains develop resistance against antimicrobial agents when they change or adapt to survive in the presence of antibiotic designed to kill them. Upon development of resistance, patient infected with MRSA require treatment with second and third choice of 
drugs which are less effective, more toxic and more expensive. ${ }^{9}$ Vancomycin against which all isolates were susceptible in this study, is the first drug of choice for the MRSA infection treatment and alternative antibiotics regimen are Clindamycin, Streptogramin, Linezolid and Daptomycin. ${ }^{11}$

Susceptibility and resistance to a wide array of $\beta$ lactam antibiotics can be deduced from the disc diffusion test result of Penicillin and Oxacillin, thus testing for other Penicillins, $\beta$ lactam $/ \beta$ lactamase inhibitors combinations, cephems and carbepenems are not required. ${ }^{7}$ Penicillin sensitive staphylococci isolates are susceptible to other penicillin, cephems and carbepenemes recommended by FDA for the treatment of $S$. aureus infection. Penicillin resistant and Oxacillin sensitive staphylococci are resistant to penicillinase labile penicillins but are susceptible to other penicillinase stable penicillins, $\beta$ lactam $/ \beta$ lactam inhibitor combinations, relevant cephems and carbepenems. Oxacillin resistant staphylococci are resistant to all currently used $\beta$ lactam antibiotics including penicillinase stable penicillins. Cefoxitin disc diffusion test can be used more efficiently as a surrogate for the detection of Oxacillin resistance in S. aureus (and S. lugdunensis and coagulase negative staphylococci) and susceptibility /or resistance to Oxacillin can be reported on the basis of Cefoxitin test result. $^{7}$
In $\beta$ lactamase test few false positive and false negative results were obtained with all the three test methods. Chromogenic test had high sensitivity and specificity as compared to other test methods. But expensiveness and local unavailability of Nitrocefin disc may impede its regular use, whereas the reagents for acidimetric and iodometric test can be prepared in house using benzyl Penicillin G. One penicillin resistant MRSA isolate that was negative with all three tests had decreased the sensitivity and specificity of these three tests. Therefore, as recommended by American Society of Microbiology 2004 for suitability of all three test methods for testing $\beta$ lactamase production in $S$. aureus and on the basis of present finding the acidimetric and iodometric test can be followed. ${ }^{6}$

\section{CONCLUSIONS}

Nosocomial MRSA strains being significantly associated with resistance to many FDA recommended antibiotics and being a major cause of nosocomial infection, such strains should be stopped from spreading to other patients. Therefore, patients infected with such strains should be identified, kept in isolation and treated with other drug of choice for MRSA. Further, healthcare workers should be trained to control hospital infection and infection control program should be conducted effectively in all health care centers.

\section{REFERENCES}

1. Hiramatsu K, Cui L, Kuroda M, Ito T. the emergence and evolution of methicillin resistant Staphylococcus aureus. Trends Microbiol. 2001;9:486-93.

2. Chambers HF. The changing epidemiology of Staphylococcus aureus. Special issue. Emerging Infect Dis. 2001;7(2):178-82.

3. Gould IM. The clinical significance of methicillin resistant Staphylococcus aureus. J Hosp Infect. 2005;61:277-82.

4. Kikuchi K. Genetic basis of neonatal methicillin resistant Staphylococcus aureus in Japan. Pediatr Int. 2003;45:223-9.

5. CDC. Laboratory diagnosis of: oxacillin/methicillin-resistant Staphylococcus aureus. [Online]. 2005 [Cited 2008 Feb 2]; Available from: URL:http://www.cdc.gov/ncidod/dhqp/ar -mrsa.html.

6. Isenberg HD. Clinical microbiology procedure handbook Volume 2. Washington (DC): American Society for Microbiology; 2004.

7. Clinical Laboratory Standards Institute. Performance standard for antimicrobial susceptibility testing: seventeenth informational supplement M100-S17. Wayne, (PA): Clinical Laboratory Standards Institute; 2007.

8. Clinical Laboratory Standards Institute. Performance standard for antimicrobial disc susceptibility tests; approved standardninth edition M2-A9. Wayne, (PA): Clinical Laboratory Standards Institute; 2006.

9. Alestig K. Tetracyclines and chloramphenicol. In: Cohen J, Powderly WG, editors. Infectious diseases. 2nd ed. Edinburgh: Mosby, Harcourt publishers limited; 2004. p. 1843-47.

10. Hsueh PR, Teng LJ, Chen WH, Pan HJ, Chen ML, Chang SC, et al. Increasing prevalence of Methicillin resistant Staphylococcus aureus causing nosocomial infections at a university hospital in Taiwan from 1986 to 2001. Antimicrobiol Agents Chemother. 2004;48:1361-4.

11. Verhoef J, Fluit AC, Scmitz FJ. Staphylococci and other micrococcaceae. In: Cohen J, Powderly WG editors. Infectious diseases. 2nd ed. Edinburgh: Mosby, Harcourt publishers limited; 2004. p. 2119-32. 